\title{
Feasibility study of the Fab fragment of a monoclonal antibody against tissue factor as a diagnostic tool
}

\author{
RYO TSUMURA ${ }^{1,2}$, RYUTA SATO ${ }^{1,2}$, FUMIAKI FURUYA ${ }^{1}$, YOSHIKATSU KOGA ${ }^{1}$, YOSHIYUKI YAMAMOTO ${ }^{3}$, \\ YUKI FUJIWARA $^{1,2}$, MASAHIRO YASUNAGA ${ }^{1}$ and YASUHIRO MATSUMURA ${ }^{1}$ \\ ${ }^{1}$ Division of Developmental Therapeutics, Exploratory Oncology Research \& Clinical Trial Center, National Cancer Center, \\ Kashiwa, Chiba 277-8577; ${ }^{2}$ Department of Integrated Biosciences, Graduate School of Frontier Sciences, \\ The University of Tokyo, Kashiwa, Chiba 277-8561; ${ }^{3}$ Department of Gastroenterology and Hepatology, \\ Institute of Clinical Medicine, Graduate School of Comprehensive Human Sciences, \\ The University of Tsukuba, Tsukuba, Ibaraki 305-8576, Japan
}

Received May 27, 2015; Accepted July 6, 2015

DOI: 10.3892/ijo.2015.3210

\begin{abstract}
Tissue factor (TF) is expressed strongly in various types of cancer, especially cancers that are often refractory to treatment, such as pancreatic cancer. In this study, we compared the differences in the biophysical and pharmacological properties of whole IgG and the Fab fragment of anti-human TF monoclonal antibody (1849 antibodies), in order to determine their suitability for application in the diagnosis and treatment of cancers. In the biophysical examination, we investigated the characteristics of 1849 -whole IgG and 1849 -Fab by SPR sensing and confocal fluorescence microscopy analysis using recombinant human TF antigen and TF-overexpressing human pancreatic cancer cell line, BxPC3, respectively. After conjugation with Alexa-Flour-647, in vivo imaging was conducted in mice bearing BxPC3 xenograft tumors. Furthermore, the distribution of the conjugates in tumors and major organs was evaluated by ex vivo study. The in vitro experiments showed that 1849 antibodies had high affinity against TF antigen.
\end{abstract}

Correspondence to: Dr Yasuhiro Matsumura, Division of Developmental Therapeutics, Exploratory Oncology Research \& Clinical Trial Center, National Cancer Center, 6-5-1 Kashiwanoha, Kashiwa, Chiba 277-8577, Japan

E-mail: yhmatsum@east.ncc.go.jp

Abbreviations: MRI, magnetic resonance imaging; PET, positron emission tomography; SPECT, single photon emission computed tomography; CT, computed tomography; mAb, monoclonal antibody; ADCs, antibody-drug conjugates; TF, tissue factor; SPR, surface Plasmon resonance; PCS, photon correlation spectroscopy; DLS, dynamic light scattering; TBR ratio, tumor-to background ratio; $\mathrm{Ka}$, the association rate constant; $\mathrm{Kd}$, the dissociation rate constant; KD, the binding affinity constant; EPR effect, enhanced permeability and retention effect

Key words: tissue factor, in vivo fluorescence imaging, antibody, Fab fragment, pancreatic cancer
In addition, 1849-Fab showed a faster dissociation rate from the antigen than 1849-whole IgG. In mice, 1849-Fab-AlexaFlour-647 showed rapid renal clearance and faster tumor accumulation, achieving a high contrast signal over nearby normal tissues in the early phase and enhanced tumor penetration after administration. On the other hand, 1849-whole IgG-Alexa-Flour-647 showed slow clearance from the blood and sustained high tumor accumulation. These results suggest that 1849 -Fab may be a useful tool for pancreatic cancer diagnosis.

\section{Introduction}

Of all clinical cancers, pancreatic cancer has one of the poorest prognoses, with an overall 5-year survival rate of $\sim 6 \%$ (1). Therefore, detection of the tumor and its micrometastases at an early stage, curative resectability of the tumor, and development of effective drugs for its treatment are desired.

In present diagnostics, several non-invasive imaging technologies, such as magnetic resonance imaging (MRI), positron emission tomography (PET), single photon emission computed tomography (SPECT) and computed tomography (CT) are available for clinical use (2). To discriminate the tumor from normal tissues, several monoclonal antibody (mAb)-based probes have been developed by exploiting tumorspecific molecules. MAbs have also been used to treat some types of cancer (e.g., malignant lymphoma, breast cancer) $(3,4)$. Moreover, antibody-drug conjugates (ADCs), by which an anticancer drug as a payload is preferentially delivered to the tumor tissue with minimal adverse effects, are under development. Thus, mAbs with high and specific affinity for tumor antigens are potentially excellent tools for cancer diagnosis and treatment.

It is well-known that cancer invasion is accompanied by an activation of blood coagulation (5). Recently, several clinical studies have revealed that cancer patients are at a high risk for the development of thrombosis (6). Hemorrhage from the tumor vessels by the invading tumor cells and subsequent fibrin clot formation to stop the bleeding occur repeatedly 
in tumor tissues (7). Moreover, this state lasts in solid tumor tissues for as long as the tumor cells survive in the body (7). Tissue factor (TF) is a $47-\mathrm{kDa}$ transmembrane glycoprotein that is known to initiate the extrinsic coagulation cascade and to play a critical role in hemostasis. Therefore, it is conceivable that high expression levels of TF may be one of the major causes of the hypercoagulable state in tumors. TF is well known to be expressed at high levels in many types of solid tumors, such as pancreatic cancer, glioma, colorectal cancer, non-small cell lung cancer, ovarian cancer, prostate cancer and breast cancer (8-10). TF is also known to be expressed in the tumor stromal cells (11-13). Some studies have indicated that activation of TF signaling in tumor cells enhances tumor growth, metastasis, inflammation and angiogenesis (14-18). Furthermore, TF expression has been shown to be correlated with a poor prognosis in patients with cancer (19-22).

We hypothesized that TF may be a promising target for imaging probes or delivery of anticancer drugs into tumor tissues, e.g., pancreatic tumor tissues. We and other groups have already reported the usefulness of anti-TF $\mathrm{mAb}$ in cancer imaging and therapy, including the Fab and $\mathrm{scFv}$ fragments (23-29). However, the optimum size as a molecular probe has not been fully evaluated from the perspective of biochemical characteristics and bio-distribution within the tumor tissue.

In the present study, we prepared an anti-TF $\mathrm{mAb}$ and its Fab fragment, and investigated their biochemical and pharmacological characteristics in vitro and in vivo, in order to determine their suitability for application in the diagnosis and treatment of cancer.

\section{Materials and methods}

Antibodies and cell line. We developed the clone 1849 rat mAb IgG2b which reacts with human TF antigen but not with mouse TF antigen. As an isotype control antibody, we also developed clone 372 rat $\mathrm{mAb}$ IgG2b which did not react with human and mouse TF antigens (30). The human pancreatic cancer cell line BxPC3 was purchased from the American Type Culture Collection (ATCC, Manassas, VA, USA). The cell line was maintained in RPMI-1640 medium (Wako, Osaka, Japan) supplemented with $10 \%$ fetal bovine serum (FBS, Gibco, Grand Island, NY, USA) and $100 \mathrm{U} / \mathrm{ml}$ of penicillin G, $100 \mu \mathrm{g} /$ $\mathrm{ml}$ of streptomycin, and $0.25 \mu \mathrm{g} / \mathrm{ml}$ of amphotericin B (Wako) in a $5 \% \mathrm{CO}_{2}$ atmosphere at $37^{\circ} \mathrm{C}$.

Generation of the Fab fragment. The 1849 and control $\mathrm{mAbs}$ were digested with papain (Worthington Biochemical, Freehold, NJ, USA) at a protein/enzyme ratio of 250:1, in a reaction buffer containing $100 \mathrm{mM}$ sodium phosphate, $2 \mathrm{mM}$ EDTA, and $10 \mathrm{mM}$ Cysteine- $\mathrm{HCl}, \mathrm{pH} 7.0$, for $2 \mathrm{~h}$ at $37^{\circ} \mathrm{C}$. The reaction buffer was then changed to $5 \mathrm{mM}$ sodium phosphate buffer ( $\mathrm{pH} 7.5$ ) for removing Cysteine-HCl using Amicon Ultra (Merck-Millipore, Darmstadt, Germany). The 1849-Fab and control-Fab were purified in a CHT ceramic hydroxyapatite column using a salt concentration gradient (Bio-Rad, Hercules, CA, USA).

Purity of the antibodies. The purity of the antibodies was evaluated by SDS-PAGE and Bioanalyzer analysis (Agilent Technologies, Santa Clara, CA, USA) under non-reducing conditions. In SDS-PAGE, the samples were loaded ( $2 \mu \mathrm{g} /$ lane) on a $4-15 \%$ polyacrylamide gradient gel (Bio-Rad). The gel was then stained with Coomassie Brilliant Blue R-250 and scanned with ChemiDoc XRS+ (Bio-Rad). The purity and molecular size of the antibodies were measured using an Agilent bioanalyzer, as prescribed in the manual, using the Agilent Protein 230 kit.

Particle size determination and surface plasmon resonance (SPR) analysis. Particle size was determined using DelsaNano HC (Beckman Coulter, Brea, CA, USA) on the basis of photon correlation spectroscopy (PCS) and dynamic light scattering (DLS). Biacore T200 (GE Healthcare, Uppsala, Sweden) was used to assess the binding of the 1849 antibodies to recombinant human TF (rhTF) antigen. Following standard amine chemistry protocols, rhTF antigen was diluted to $1 \mu \mathrm{g} / \mathrm{ml}$ in $10 \mathrm{mM}$ sodium acetate buffer $(\mathrm{pH}$ 5.0) and immobilized on the CM5 sensor chips (GE Healthcare) at lower density (17.7 resonance units) to only allow monovalent binding. We used the single-cycle kinetics to measure the affinity of the antibodies. Antibodies in PBS were passed over the sensor chips, and the interactions were monitored for $30 \mathrm{sec}$. The sensor surface was washed with PBS to detect dissociation and then regenerated with $10 \mathrm{mM}$ Glycine- $\mathrm{HCl}(\mathrm{pH} 1.5)$ at the end of each experiment.

Antibody labeling. The 1849-whole IgG, 1849-Fab, controlwhole IgG and control-Fab were chemically labeled with the fluorescent dyes Alexa-Fluor-647 (AF647, Invitrogen, Eugene, OR, USA), according to the manufacturer's protocol (MP 00143, Amine-Reactive Probes, Invitrogen). Following the labeling reaction, the antibody concentration and degree of labeling were measured by determining the absorbance values at $280 \mathrm{~nm}$ and $650 \mathrm{~nm}$ with a Nano Drop ND-1000 spectrometer (Thermo Fisher Scientific, Wilmington, DE, USA).

The final concentration of the antibodies-AF647 was $1.0 \mathrm{mg} / \mathrm{ml}$, and the final dye molarities of 1849 -whole IgG-AF647, 1849-Fab-AF647, control-whole IgG-AF647 and control-Fab-AF647 were 19.5, 18.2, 21.9 and $21.5 \mu \mathrm{M}$, respectively.

Confocal fluorescence microscopy analysis. BxPC3 cells were planted on BD Falcon 4-well chamber slide at $1 \times 10^{5}$ cells/well. After $24 \mathrm{~h}$ of incubation, cells were incubated with $500 \mu \mathrm{l}$ of RPMI medium containing $0.1 \mu \mathrm{M} 1849$-whole IgG-AF647, 1849-Fab-AF647, control-whole IgG-AF647 or controlFab-AF647 for $1 \mathrm{~h}$ at $37^{\circ} \mathrm{C}$. Cells were washed three times with PBS, then fixed with $4 \%$ paraformaldehyde in PBS for $15 \mathrm{~min}$ at RT and stained with DAPI solution $(1 \mu \mathrm{g} / \mathrm{ml})$ for $5 \mathrm{~min}$ at RT. The slides were covered with Fluoromount-G (SouthernBiotech, Birmingham, AL, USA). Fluorescence images were obtained with a fluorescence microscope, BIOREVO BZ9000 (Keyence, Osaka, Japan).

Flow cytometric analysis. The cell-binding activities of the antibodies-AF647 to BxPC3 cells were evaluated by flow cytometry. Briefly, BxPC3 cells were harvested and suspended in PBS with $0.5 \%$ bovine serum albumin (BSA, Wako) and $2 \mathrm{mM}$ EDTA (B.E.PBS) at a density of $4 \times 10^{5}$ cells $/ \mathrm{ml}$. The BxPC3 cells were then incubated with the antibodies-AF647 
Table I. Particle size and binding parameters of antibodies.

\begin{tabular}{lcccc}
\hline & Particle size $(\mathrm{nm})$ & KD $(\mathrm{M})$ & Ka $(1 / \mathrm{Ms})$ & Kd $(1 / \mathrm{s})$ \\
\hline 1849 whole IgG & $13.1 \pm 4.6$ & $3.665 \times 10^{-10}$ & $3.770 \times 10^{4}$ & $1.382 \times 10^{-5}$ \\
1849 Fab & $5.0 \pm 2.4$ & $2.001 \times 10^{-9}$ & $2.895 \times 10^{4}$ & $5.795 \times 10^{-5}$ \\
Control whole IgG & $13.0 \pm 4.8$ & N/A & N/A & N/A \\
Control Fab & $5.5 \pm 1.8$ & N/A & N/A & N/A \\
\hline
\end{tabular}

The particle size is shown as the mean \pm standard deviation. N/A showed no data.

at each concentration $(1,10$ and $100 \mu \mathrm{M})$ for $30 \mathrm{~min}$ on ice, and washed three times with B.E.PBS. Subsequently, propidium iodide $(0.4 \mu \mathrm{l} / \mathrm{ml}$; PI, Invitrogen) was added, and the cell binding activities were analyzed with the guava easyCyte (Millipore) using FlowJo analysis software (Tree Star Inc., Ashland, OR, USA). As a negative control, we added the fraction containing PI and not the antibodies-AF647.

Animal models. Four-week-old female BALB/c nude mice were purchased from SLC Japan (Shizuoka, Japan). A week later, the mice were inoculated subcutaneously in the right flank with $1 \times 10^{6}$ BxPC3 cells suspended in $100 \mu 1$ PBS. The test antibodies were injected intravenously into the tail vein of the mice when the tumor volume reached $\sim 100-150 \mathrm{~mm}^{3}$, as measured with calipers, and calculated by the formula: volume $=$ length $\times(\text { width })^{2} \times 1 / 2$. All animal procedures and experiments were conducted with the approval of the Committee for Animal Experimentation of the National Cancer Center, Japan. These guidelines meet the ethical standards required by law and also comply with the guidelines for the use of experimental animals in Japan.

In vivo and ex vivo fluorescence imaging. In vivo and ex vivo fluorescence imaging were performed using IVIS Kinetic imaging system and analyzed using IVIS Living Imaging 3.0 software (Caliper Life Sciences, Hopkinton, MA, USA). A filter set (excitation at $605 \mathrm{~nm}$, emission at $640 \mathrm{~nm}$ ) was used for acquiring the fluorescence of the AF647 conjugated antibodies. All fluorescence images were acquired in identical illumination settings and normalized as photons per second per centimeter square per steradian $\left(\mathrm{p} / \mathrm{s} / \mathrm{cm}^{2} / \mathrm{sr}\right)$. Quantitative data were obtained from ROI (regions of interest) analysis of the fluorescence images. The mice were injected with $\sim 100 \mu \mathrm{g}$ of antibodies-AF647 via the tail vein in both studies.

In vivo imaging was performed at 1,2, 3, 6, 9, 12, 24 and $72 \mathrm{~h}$ post-injection. The mean fluorescence intensity of the left flank region, on the side contralateral to the tumor, was used as the background value in order to investigate the tumor-to-background ratio (TBR) of 1849-whole IgG-AF647 and 1849-Fab-AF647. The TBR was determined as the mean fluorescence intensity of the tumor divided by the mean background intensity. Statistical analysis were performed using Student's t-test. P-values of $<0.05$ and $<0.01$ were considered statistically significant. In the ex vivo study, the mice were euthanized at 1, 3, 6, 24 and $72 \mathrm{~h}$ post-injection, followed by excision of the tumor and major organs from the mice. The tumor and the major organs, excluding blood, were washed with PBS and placed on a dish with no fluorescence intensity in the measurement range. The mean fluorescence intensity of the tumor and major organs obtained from the mice which were not injected with antibodies was used as the background value.

Distribution of antibodies. In the distribution study of antibodies, the mice were injected with $\sim 300 \mu \mathrm{g}$ of 1849 -whole IgG-AF647 and 1849-Fab-AF647 via the tail vein. The mice were euthanized under deep anesthesia at $3 \mathrm{~h}$ after the injection, and the tumors were excised. The tumors were then embedded in Tissue-Tec optimal-cutting-temperature compound (Sakura Finetek, Tokyo, Japan) and frozen at $-80^{\circ} \mathrm{C}$ until use. Frozen sections, $6-\mu \mathrm{m}$-think, of the tumors were fixed with $4 \%$ paraformaldehyde in PBS for $15 \mathrm{~min}$ at RT and blocked with 5\% skim milk in PBS for $1 \mathrm{~h}$ at RT. The sections were then incubated with goat anti-mouse CD31 polyclonal antibody $(2 \mu \mathrm{g} / \mathrm{ml}$; R\&D Systems, Minneapolis, MN, USA) for $1 \mathrm{~h}$ at RT, and then with AF555 conjugated anti-goat polyclonal antibody ( $4 \mu \mathrm{g} / \mathrm{ml}$; Invitrogen) for $1 \mathrm{~h}$ at RT. Subsequently, the sections were stained with DAPI solution $(1 \mu \mathrm{g} / \mathrm{ml})$ for $5 \mathrm{~min}$ at RT and the slides were covered with Fluoromount-G. Fluorescence images were obtained with BIOREVO BZ9000.

\section{Results}

Generation and purity of the antibodies. Fig. 1A shows a schematic diagram of production of the Fab fragment from the whole IgG. The Fab fragments were visualized as an almost single band on SDS-PAGE, which indicated the high purity of the fragments (Fig. 1B). The results of the bioanalyzer analysis also confirmed the high purity (Fig. 1C-F).

Biochemical characteristics of the antibodies. The particle sizes of the antibodies were correlated with their molecular weights (Table I). There were no significant differences in the molecular weight between 1849 -whole IgG and control-whole IgG, or 1849-Fab and control-Fab. In the SPR analysis, while the association rate constant $(\mathrm{Ka})$ values of 1849 -whole $\mathrm{IgG}$ and 1849 -Fab were almost the same, the dissociation rate constant $(\mathrm{Kd})$ value of 1849 -Fab was $\sim 4.2$-fold higher than that of 1849-whole IgG (Table I). Consequently, the binding affinity constant (KD) value of 1849-Fab was $\sim 5.5$-fold higher than that of the 1849 -whole $\operatorname{IgG}$ because of high dissociation. 

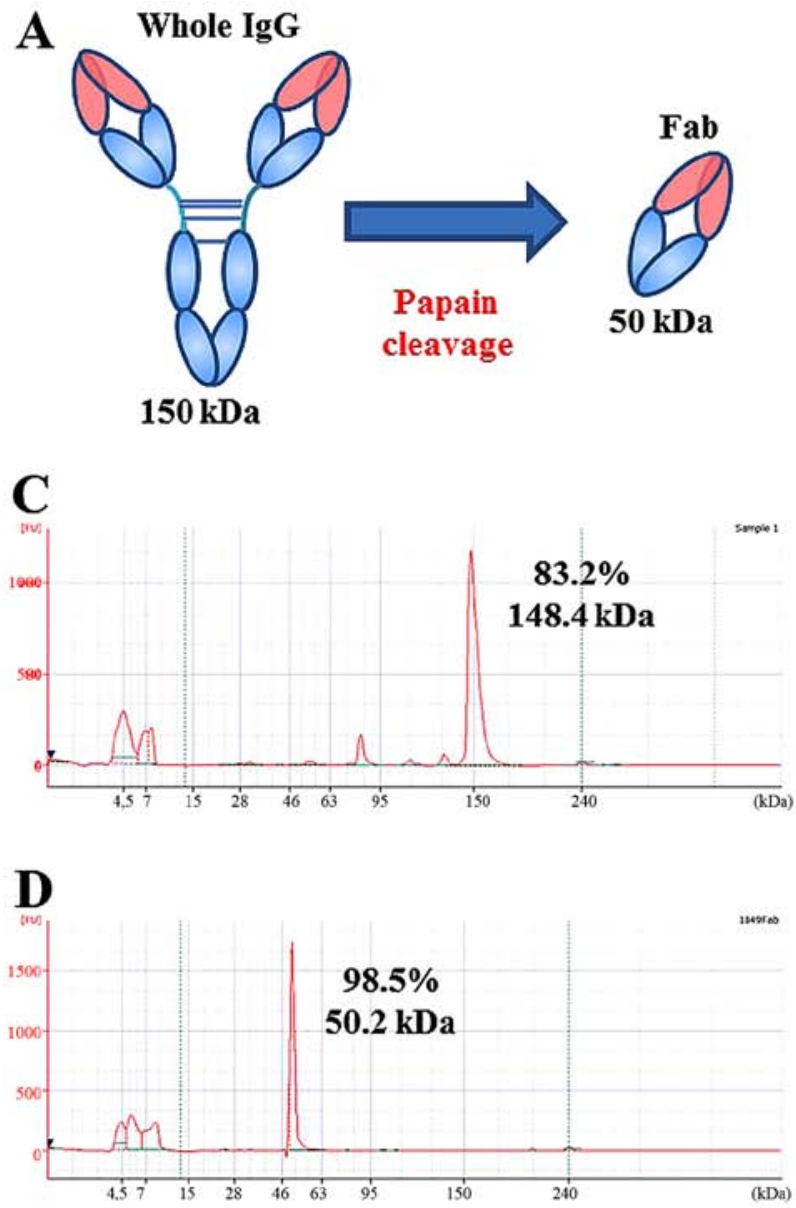

B
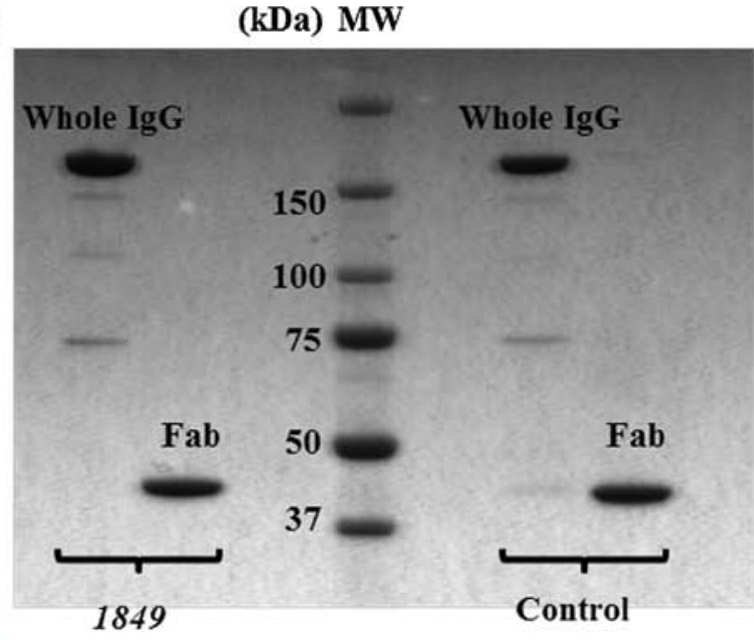

$\mathbf{E}$
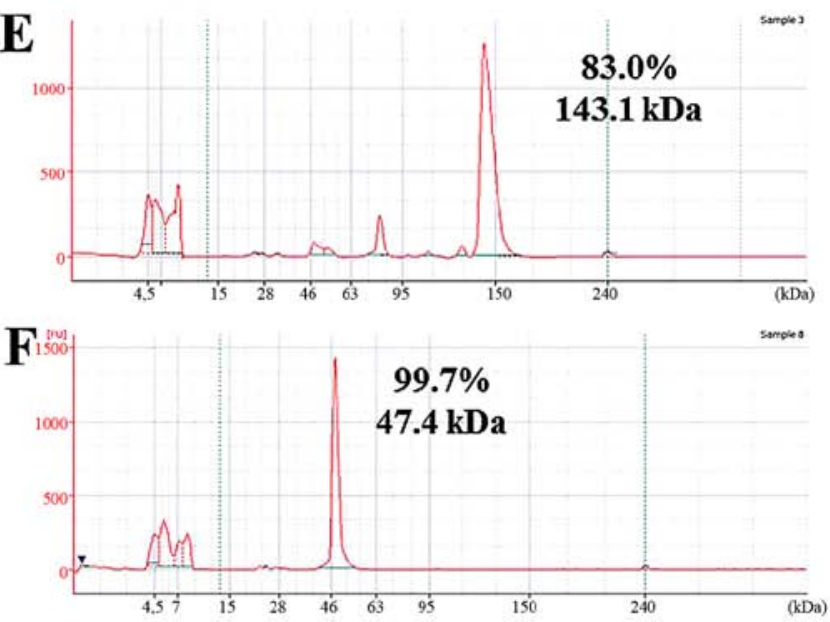

Figure 1. Generation and purity of antibodies. (A) Diagram of papain cleavage. (B) SDS-PAGE of antibodies under non-reducing conditions. The purities and molecular weights of (C) 1849-whole IgG and (D) 1849-Fab, (E) control-whole IgG, (F) control-Fab, were determined by bioanalyzer analysis. The three peaks of low molecular size $(\sim 10 \mathrm{kDa})$ denote the system peaks and the marker peak.
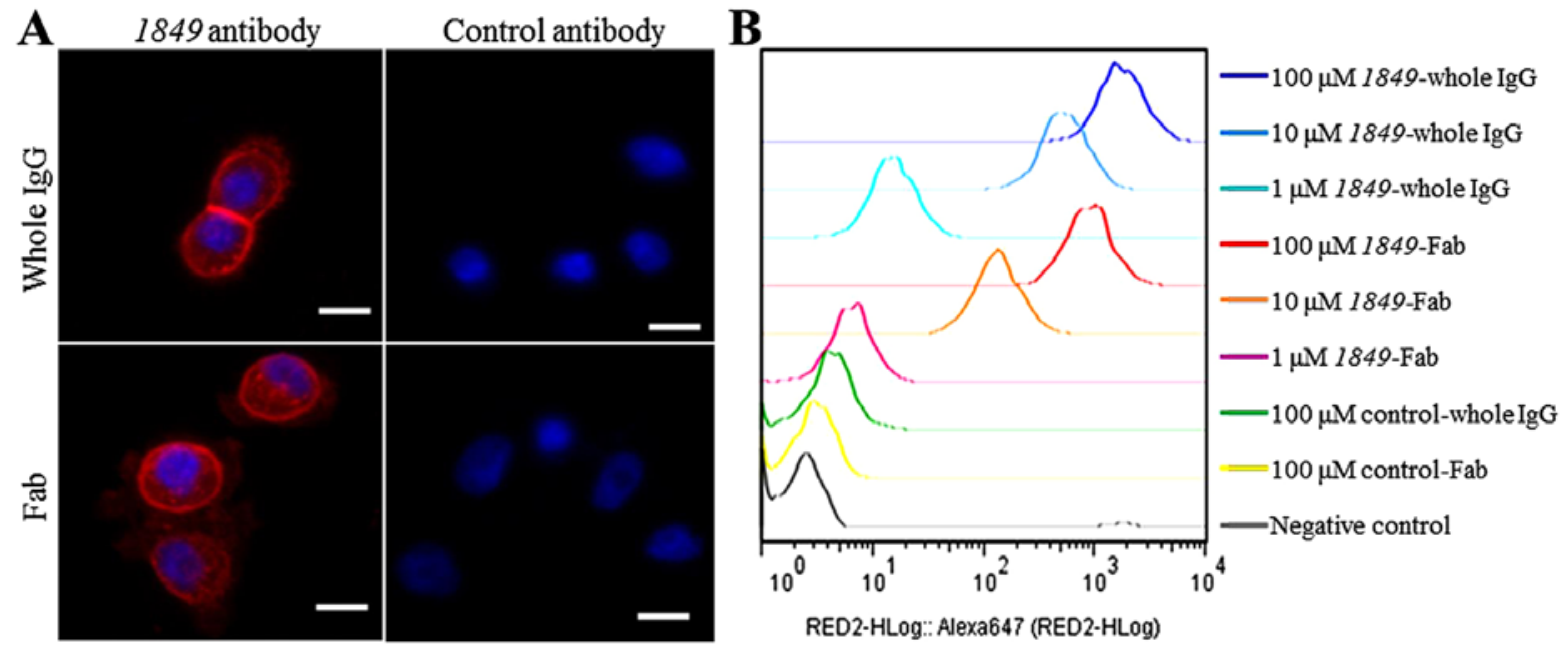

Figure 2. Confocal fluorescence microscopy analysis and flow cytometric analysis. (A) After incubation with fluorescence labeled antibodies for $1 \mathrm{~h}$, BxPC3 cells were observed by a fluorescence microscope. The red and blue colors indicate antibodies-AF647 and nuclei, respectively. Scale bar, $10 \mu \mathrm{m}$. (B) The cellbinding activities of the 1849 antibodies-AF647 and control antibodies-AF647 at each concentration were evaluated using BxPC 3 cells.

Confocal fluorescence microscopy analysis revealed that 1849-whole IgG-AF647 and 1849-Fab-AF647 bound to the membrane of BxPC3 cells and internalized into cells through TF antigen on the membrane (Fig. 2A). On the other hand, 

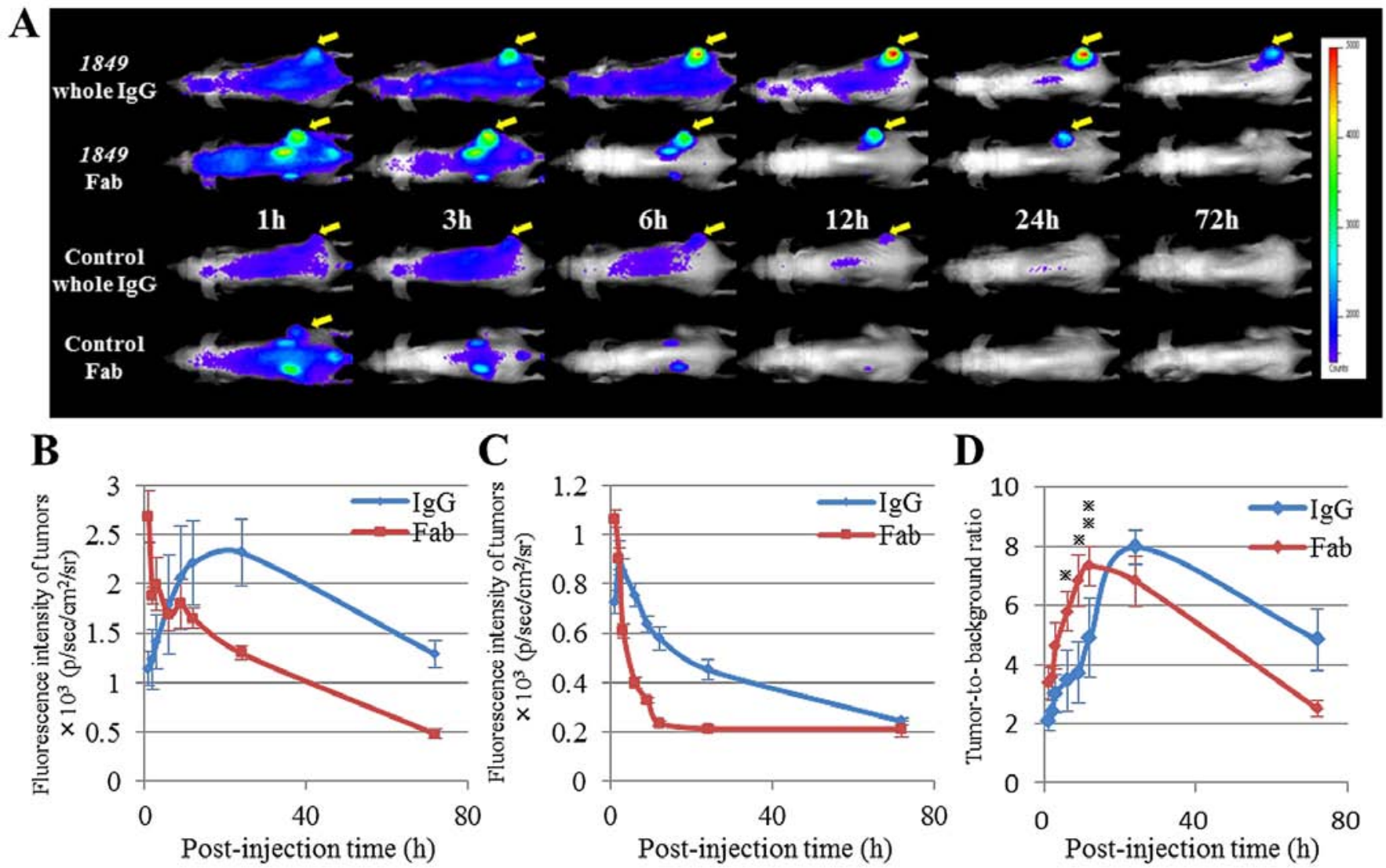

D

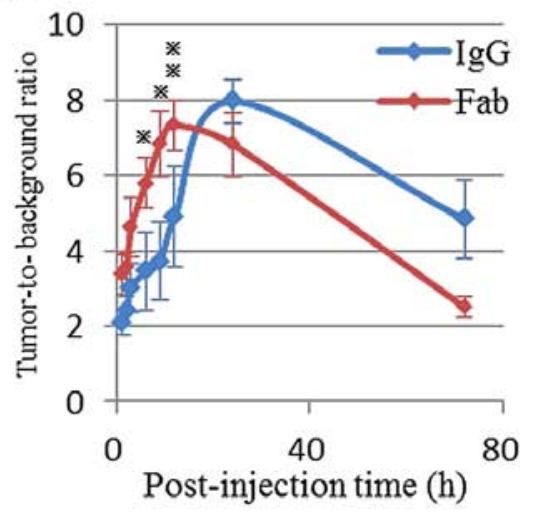

Figure 3. The tumor targeting efficacy of antibodies by multiple time-point in vivo fluorescence imaging in BxPC3-tumor bearing mice ( $\mathrm{n}=3$ ). (A) Representative fluorescence images. Fluorescence intensity of the tumors at all the time-points post injection for (B) the 1849 antibodies and (C) the control antibodies. (D) Tumor-to-background ratios were calculated as the mean fluorescence intensity of the tumor divided by the background fluorescence intensity. The mean fluorescence intensity of the left flank, on the side contralateral to the tumor, was used as the background value. All data in (B-D) are presented as mean \pm standard error. Arrows show the tumor regions. The P-value was calculated with Student's t-test. ${ }^{*} \mathrm{P}<0.05$. $^{* *} \mathrm{P}<0.01$.

control-whole IgG-AF647 and control-Fab-AF647 did not bind to the BxPC3 cells. Flow cytometric analysis confirmed a high affinity of 1849-antibodies-AF647 to BxPC3 cells depending on the concentration (Fig. 2B).

In vivo imaging. The biodistribution and tumor targeting efficacy of each of the antibodies-AF647 were measured in mice bearing BxPC3 tumors by our in vivo imaging system (Fig. 3A-C). At all time-points, both 1849-whole IgG-AF647 and 1849-Fab-AF647 showed notably higher tumor accumulation than control antibodies-AF647. The tumor accumulation of 1849-whole IgG-AF647 reached its peak at $24 \mathrm{~h}$ after the injection, whereas that of 1849-Fab-AF647 reached its maximum at $1 \mathrm{~h}$ after the injection. Both 1849-Fab-AF647 and control-Fab-AF647 showed high distribution into the kidneys in the early phase. Based on these fluorescence images, the TBR values of 1849-whole IgG-AF647 and 1849-Fab-AF647 were determined at all the time-points examined (Fig. 3D). The TBRs of 1849-whole IgG-AF647 and 1849-Fab-AF647 reached their maximum at 24 and $12 \mathrm{~h}$ after the injection, respectively. The TBR of 1849-Fab-AF647 was higher than that of 1849-whole IgG-AF647 at any time-points within $12 \mathrm{~h}$ after the injection ( 3 and $6 \mathrm{~h}, \mathrm{P}<0.05 .9 \mathrm{~h}, \mathrm{P}<0.01)$. On the other hand, the TBR of 1849-whole IgG-AF647 was higher than that of 1849-Fab-AF647 at any time-points after $24 \mathrm{~h}$ (Fig. 3D).

Ex vivo studies. The accumulation of antibodies-AF647 into the tumors was almost consistent with those observed by in vivo fluorescence imaging (Fig. 4A). The blood, liver and heart, the tissue-to-background ratios of 1849-whole IgG-AF647 were higher than those of 1849-Fab-AF647 (Fig. 4B, C and G), while the kidney tissue-to-background ratio was notably higher for 1849-Fab-AF647 than for 1849-whole IgG-AF647 at all timepoints (Fig. 4D). Moreover, the uptake and distribution of these antibodies in other normal tissues such as the lung, spleen and muscle were significantly low (Fig. 4E, F and H).

Distribution of the antibodies in the tumor tissues. To analyze the intratumoral distribution of 1849-whole IgG-AF647 and 1849-Fab-AF647, immunofluorescence staining was performed (Fig. 5). At $3 \mathrm{~h}$ after the injection, the fluorescence intensity of 1849-Fab-AF647 in the BxPC3 tumor tissue was higher than that of 1849-whole IgG-AF647. This result was consistent with both the results of the in vivo fluorescence imaging and ex vivo study. In addition, there was a clear difference in intratumoral distribution between 1849-whole IgG-AF647 and 1849Fab-AF647. 1849-whole IgG-AF647 was localized mainly in 

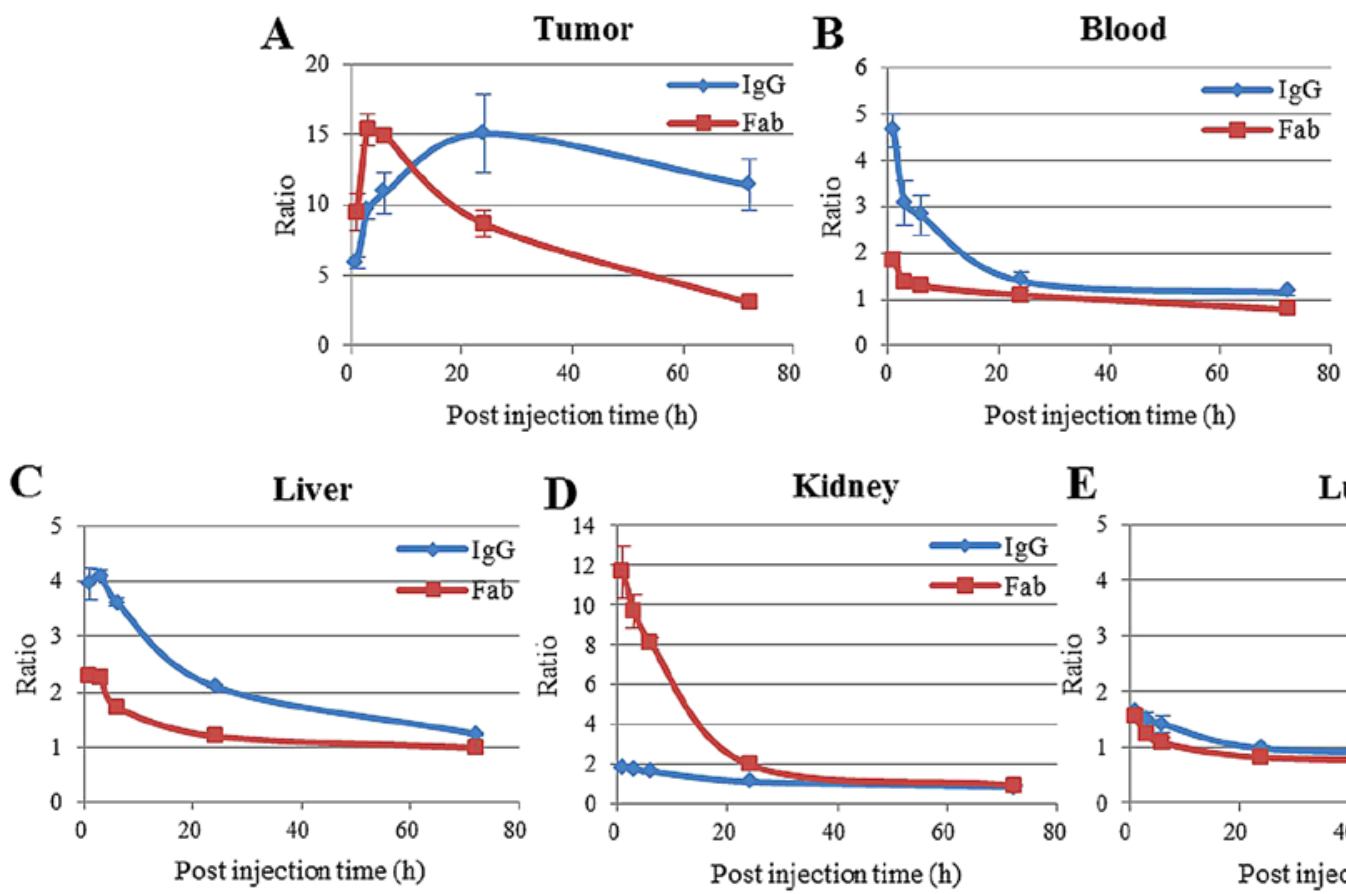

E Lung
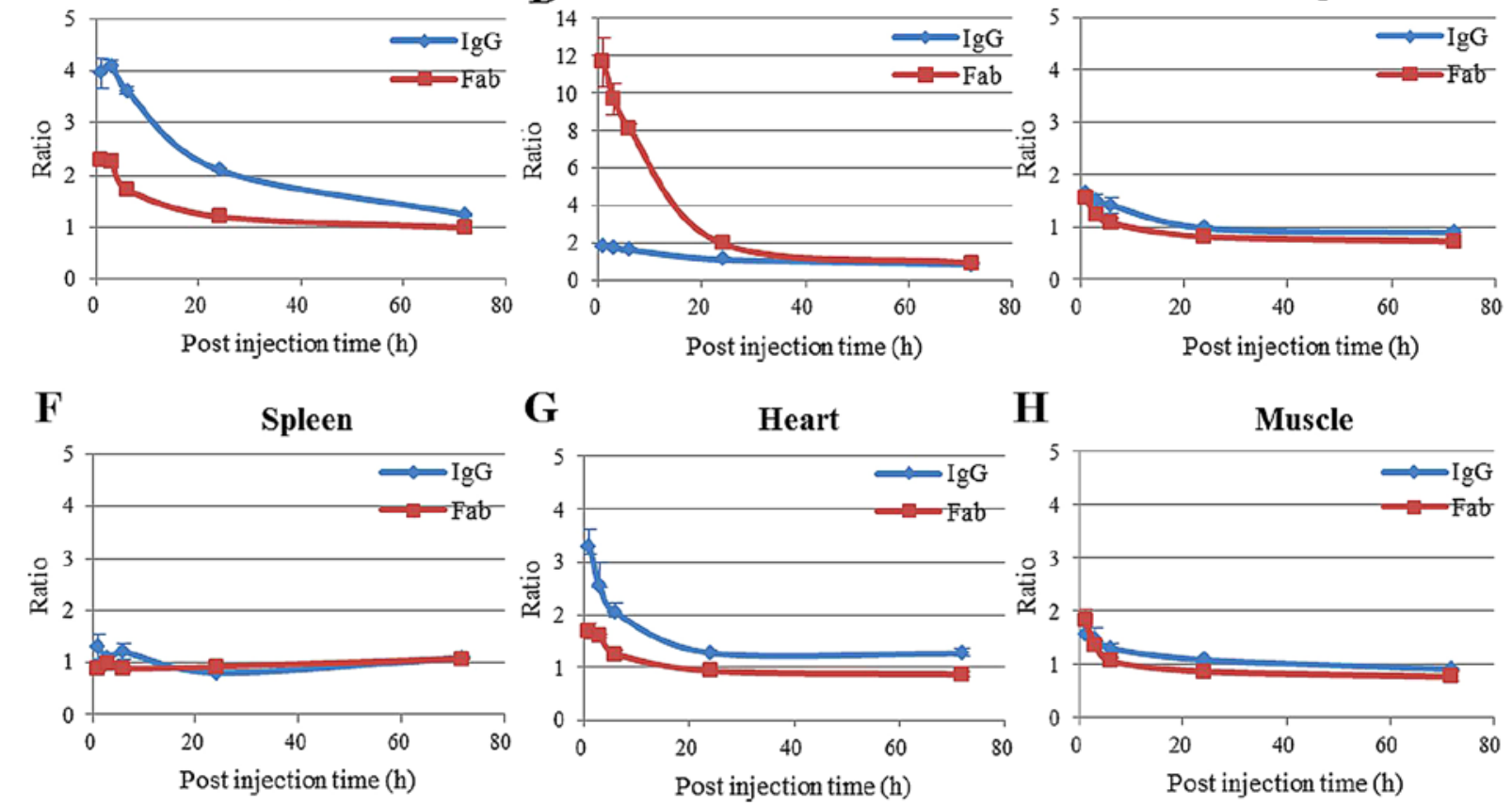

Figure 4. Accumulation of 1849 -whole IgG and 1849 -Fab in the tumor and major organs $(\mathrm{n}=3)$. As control values, the mean fluorescence intensities of the tumor and major organs of mice that were not injected with antibodies, were used. All data are presented as mean \pm standard error.

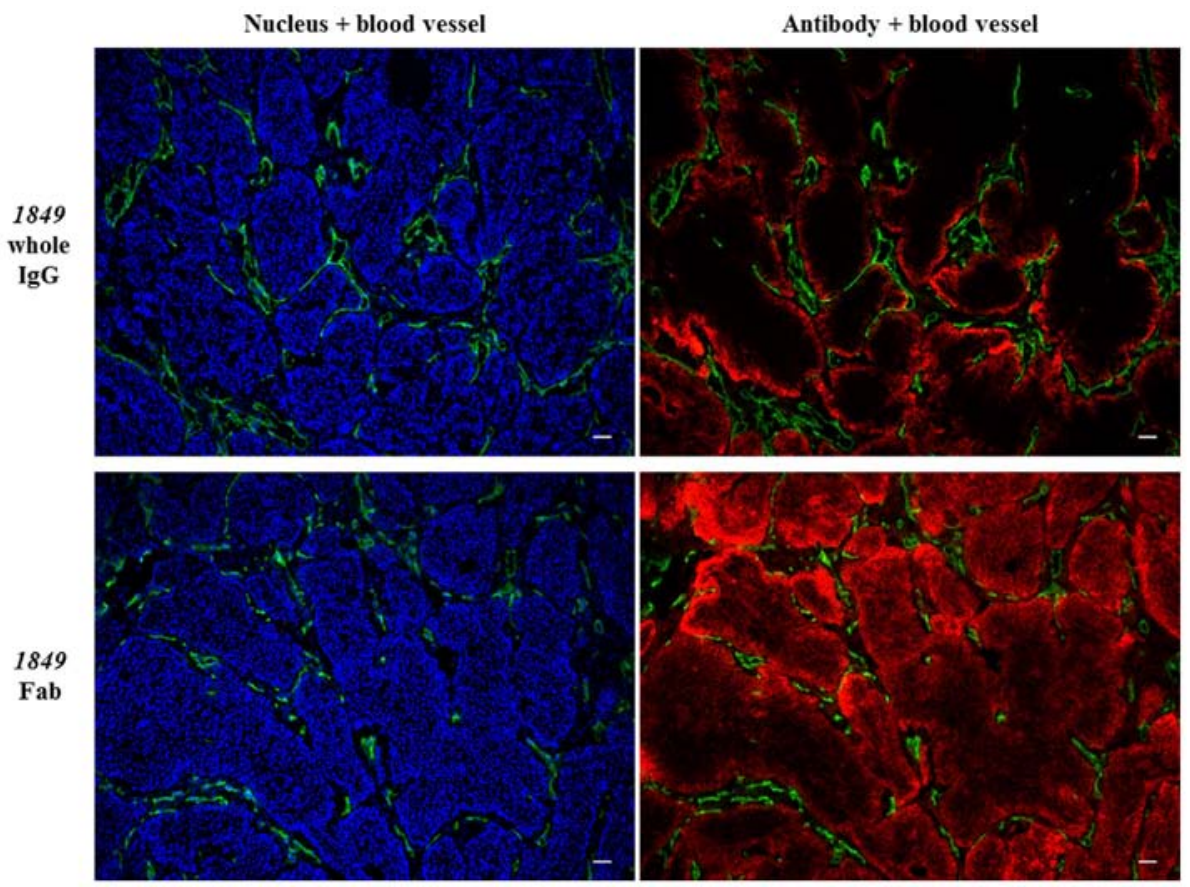

Figure 5. Distribution of the 1849 -whole IgG and 1849 -Fab in the BxPC3 tumor tissues. This study was performed by immunofluorescence staining at $3 \mathrm{~h}$ post-injection and analyzed under the same conditions. Scale bars indicate $50 \mu \mathrm{m}$. The red, blue and green colors indicate the injected antibodies, nuclei and blood vessels, respectively. 
the periphery of the tumor cell clusters, while 1849-Fab-AF647 penetrated into the center of the tumor clusters.

\section{Discussion}

A large body of basic and clinical studies has shown overexpression of TF in various types of solid tumor tissues (8-10). We hypothesized that TF might therefore be a promising target for cancer diagnosis and therapy and developed a rat $\mathrm{mAb}$ (clone 1849) against human TF.

The purpose of this study was to characterize 1849-whole IgG and 1849-Fab in vitro and in vivo, in order to clarify the suitability of their application for diagnostic or therapeutic purpose, which would enhance the potential of their application to clinical studies. We used a mouse xenograft model of human pancreatic cancer as the TF-overexpressing tumor.

First, SPR analysis showed that 1849 -Fab could dissociate from antigen more rapidly than 1849-whole IgG (Table I). Although rhTF antigen was coated to the chip at lower density, this result is explained by the presence in 1849-whole IgG of two antigen-binding sites, so one Fab-arm can still bind the antigen even if the other is detached, in contrast to only one site in 1849-Fab. Confocal fluorescence microscopy analysis and Flow cytometric analysis showed that 1849-antibodiesAF647 had a high affinity and specificity to the TF antigen expressed in BxPC3 cells.

In the in vivo imaging study, the 1849 antibodies-AF647 showed higher accumulation in the tumors at all-time-points as compared to control antibodies having passive targeting which depends on the enhanced permeability and retention (EPR) effect (31). It was clear that the EPR effect combined with the active targeting against human TF antigen contributed to the high tumor accumulation of 1849 antibodies as compared to that of control isotype IgG. Furthermore, the in vivo imaging showed that the kinetics of whole $\mathrm{IgG}$ and Fab changed due to the differences of their molecular sizes and affinities. In the presence of the Fc domain, both 1849whole IgG-AF647 and control-whole IgG-AF647 showed prolonged systemic circulation in the body (32). The property of IgG of having a high molecular weight (150 kDa), above the renal clearance threshold of $\sim 65 \mathrm{kDa}$, permits it to escape renal clearance, which facilitates prolonged circulation (33). In the imaging using whole IgG, the prolonged circulation time causes a sustained high background signal. On the other hand, because Fab lacks the Fc domain and is smaller in size $(\sim 50 \mathrm{kDa})$, Fab can pass through the renal glomeruli, resulting in rapid clearance from the body and a low background signal. Moreover, the tumor signal intensity of 1849-Fab-AF647 appeared to reach its peak immediately after the injection, whereas that of 1849-whole IgG-AF647 reached its peak at about $20 \mathrm{~h}$ after the injection. These properties led to the differences in the TBR between 1849-whole IgG-AF647 and 1849-Fab-AF647, namely, a maximum peak of 1849-whole IgG-AF647 at $24 \mathrm{~h}$ and of 1849-Fab-AF647 at $12 \mathrm{~h}$ after the injection. The results of the in vivo imaging were confirmed by the results of the ex vivo study. In brief, 1849-Fab-AF647 showed rapid tumor accumulation and rapid blood clearance, whereas in contrast, 1849-whole IgG-AF647 showed sustained high tumor accumulation and prolonged circulation in the blood. This study also showed that 1849-whole
IgG-AF647 and 1849-Fab-AF647 were mainly excreted by the liver and kidney, respectively.

The present immunofluorescence staining study showed that 1849 -Fab-AF647 penetration was deeper into the tumors than 1849-whole IgG-AF647. It was clear that the smaller size of 1849-Fab-AF647 allowed more efficient tumor penetration. In the histological examination, the BxPC3 cells at the tumor front showed stronger TF expression than those in the central region (30). Because the 1849-whole IgG-AF647 with its high affinity for the antigen may be trapped and kept by TF-overexpressing cells at the tumor front, it hardly penetrates into the tumor center at the early phase. On the other hand, 1849-Fab-AF647, with its fast dissociation rate and low internalization efficiency, may be able to penetrate the tumor center more easily (34). Although it is recognized that homogeneous penetration of the imaging probe is less important than achieving a high TBR value (35), improved tumor penetration would increase the sensitivity of detection of small-sized tumors or micrometastases.

In conclusion, the properties of $1849-\mathrm{Fab}$, namely, fast dissociation from TF antigen, rapid tumor accumulation, efficient tumor penetration, and rapid body clearance make it potentially suitable for diagnostic applications. A diagnostic test with the Fab probe would provide a high contrast and fine tumor imaging within half a day. Fab can also minimize several of the adverse effects (e.g., hypersensitivity) of whole IgG. In addition, it allows use of a short-half-life radionuclide (e.g., ${ }^{99 \mathrm{~m}} \mathrm{Tc}$, half-life $6 \mathrm{~h}$ ) (36), which can shorten the scan time and reduce the total body radiation dose. These features would benefit both outpatients and healthy people as the target populations for diagnostic application. On the other hand, the properties of 1849-whole IgG, namely, high avidity, high tumor accumulation and prolonged biological half-life, make it more suitable for therapeutic applications. We thus propose the importance of the developmental strategy of selecting a suitable antibody depending on whether the intended application is diagnostic or therapeutic. For clinical application of our 1849 antibodies, further investigation, i.e., PET or SPECT imaging for diagnostic application and ADC for therapeutic application using an appropriate experimental tumor model with high TF expression and abundant tumor stroma, is needed.

\section{Acknowledgements}

This study was supported by a Grant-in-Aid from the Japan Society for the Promotion of Science (JSPS) through the Funding Program for World-Leading Innovative R\&D on Science and Technology (FIRST Program) initiated by the Council for Science and Technology Policy (CSTP) (to Y. Matsumura) and the National Cancer Center Research and Development Fund (23-A-45 to Y. Matsumura). We thank Mrs. M. Kanzaki for supporting the animal experiments and Ms. M. Nakayama for secretarial help.

\section{References}

1. Howlader N, Noone AM, Krapcho M, Garshell J, Neyman N, Altekruse SF, Kosary CL, Yu M, Ruhl J, Tatalovich Z, et al: National Cancer Institute: SEER Cancer Statistics Review, 1975-2010. http://seer.cancer.gov/csr/1975_2010/. Accessed June 14, 2013. 
2. Kaur S, Venktaraman G, Jain M, Senapati S, Garg PK and Batra SK: Recent trends in antibody-based oncologic imaging. Cancer Lett 315: 97-111, 2012.

3. Verma S, Miles D, Gianni L, Krop IE, Welslau M, Baselga J, Pegram M, Oh DY, Diéras V, Guardino E, et al; EMILIA Study Group: Trastuzumab emtansine for HER2-positive advanced breast cancer. N Engl J Med 367: 1783-1791, 2012.

4. Ricart AD and Tolcher AW: Technology insight: Cytotoxic drug immunoconjugates for cancer therapy. Nat Clin Pract Oncol 4 245-255, 2007.

5. Rickles FR, Levine M and Edwards RL: Hemostatic alterations in cancer patients. Cancer Metastasis Rev 11: 237-248, 1992.

6. Stein PD, Beemath A, Meyers FA, Skaf E, Sanchez J and Olson RE: Incidence of venous thromboembolism in patients hospitalized with cancer. Am J Med 119: 60-68, 2006.

7. Matsumura Y: Cancer stromal targeting (CAST) therapy. Adv Drug Deliv Rev 64: 710-719, 2012.

8. Callander NS, Varki N and Rao LV: Immunohistochemical identification of tissue factor in solid tumors. Cancer 70: 11941201, 1992.

9. Kasthuri RS, Taubman MB and Mackman N: Role of tissue factor in cancer. J Clin Oncol 27: 4834-4838, 2009.

10. van den Berg YW, Osanto S, Reitsma PH and Versteeg HH: The relationship between tissue factor and cancer progression: Insights from bench and bedside. Blood 119: 924-932, 2012.

11. Contrino J, Hair G, Kreutzer DL and Rickles FR: In situ detection of tissue factor in vascular endothelial cells: Correlation with the malignant phenotype of human breast disease. Nat Med 2: 209-215, 1996

12. Vrana JA, Stang MT, Grande JP and Getz MJ: Expression of tissue factor in tumor stroma correlates with progression to invasive human breast cancer: Paracrine regulation by carcinoma cell-derived members of the transforming growth factor beta family. Cancer Res 56: 5063-5070, 1996.

13. Ueno T, Toi M, Koike M, Nakamura S and Tominaga T: Tissue factor expression in breast cancer tissues: Its correlation with prognosis and plasma concentration. Br J Cancer 83: 164-170, 2000 .

14. Versteeg $\mathrm{HH}$ and Ruf W: Emerging insights in tissue factordependent signaling events. Semin Thromb Hemost 32: 24-32, 2006.

15. Langer $\mathrm{F}$ and Bokemeyer $\mathrm{C}$ : Crosstalk between cancer and haemostasis. Implications for cancer biology and cancer-associated thrombosis with focus on tissue factor. Hamostaseologie 32: 95-104, 2012

16. Khorana AA, Ahrendt SA, Ryan CK, Francis CW, Hruban RH, Hu YC, Hostetter G, Harvey J and Taubman MB: Tissue factor expression, angiogenesis, and thrombosis in pancreatic cancer. Clin Cancer Res 13: 2870-2875, 2007

17. Milsom CC, Yu JL, Mackman N, Micallef J, Anderson GM, Guha A and Rak JW: Tissue factor regulation by epidermal growth factor receptor and epithelial-to-mesenchymal transitions: Effect on tumor initiation and angiogenesis. Cancer Res 68: 10068-10076, 2008

18. Hjortoe GM, Petersen LC, Albrektsen T, Sorensen BB, Norby PL, Mandal SK, Pendurthi UR and Rao LV: Tissue factor-factor VIIaspecific up-regulation of IL-8 expression in MDA-MB-231 cells is mediated by PAR-2 and results in increased cell migration. Blood 103: 3029-3037, 2004

19. Regina S, Valentin JB, Lachot S, Lemarié E, Rollin J and Gruel Y: Increased tissue factor expression is associated with reduced survival in non-small cell lung cancer and with mutations of TP53 and PTEN. Clin Chem 55: 1834-1842, 2009.

20. Yamashita H, Kitayama J, Ishikawa $M$ and Nagawa H: Tissue factor expression is a clinical indicator of lymphatic metastasis and poor prognosis in gastric cancer with intestinal phenotype. J Surg Oncol 95: 324-331, 2007.
21. Kaido T, Oe H, Yoshikawa A, Mori A, Arii S and Imamura M: Tissue factor is a useful prognostic factor of recurrence in hepatocellular carcinoma in 5-year survivors. Hepatogastroenterology 52: 1383-1387, 2005

22. Nitori N, Ino Y, Nakanishi Y, Yamada T, Honda K, Yanagihara K, Kosuge T, Kanai Y, Kitajima M and Hirohashi S: Prognostic significance of tissue factor in pancreatic ductal adenocarcinoma. Clin Cancer Res 11: 2531-2539, 2005.

23. Sato R, Obonai T, Tsumura R, Tsumoto K, Koga Y, Yasunaga M and Matsumura Y: Preparation and characterization of antitissue factor single-chain variable fragment antibody for cancer diagnosis. Cancer Sci 105: 1631-1637, 2014.

24. Koga Y, Manabe S, Aihara Y, Sato R, Tsumura R, Iwafuji H, Furuya F, Fuchigami H, Fujiwara Y, Hisada Y, et al: Antitumor effect of antitissue factor antibody-MMAE conjugate in human pancreatic tumor xenografts. Int J Cancer: Feb 20, 2015 (Epub ahead of print). doi: $10.1002 /$ ijc. 29492 .

25. Hong H, Zhang Y, Nayak TR, Engle JW, Wong HC, Liu B, Barnhart TE and Cai W: Immuno-PET of tissue factor in pancreatic cancer. J Nucl Med 53: 1748-1754, 2012.

26. Versteeg HH, Schaffner F, Kerver M, Petersen HH, Ahamed J, Felding-Habermann B, Takada Y, Mueller BM and Ruf W: Inhibition of tissue factor signaling suppresses tumor growth. Blood 111: 190-199, 2008.

27. Harter PN, Dützmann S, Drott U, Zachskorn C, Hattingen E, Capper D, Gessler F, Senft C, Seifert V, Plate KH, et al: Anti-tissue factor (TF9-10H10) treatment reduces tumor cell invasiveness in a novel migratory glioma model. Neuropathology 33: 515-525, 2013

28. Breij EC, de Goeij BE, Verploegen S, Schuurhuis DH, Amirkhosravi A, Francis J, Miller VB, Houtkamp M, Bleeker WK, Satijn D, et al: An antibody-drug conjugate that targets tissue factor exhibits potent therapeutic activity against a broad range of solid tumors. Cancer Res 74: 1214-1226, 2014.

29. Shi S, Hong H, Orbay H, Graves SA, Yang Y, Ohman JD, Liu B, Nickles RJ, Wong HC and Cai W: ImmunoPET of tissue factor expression in triple-negative breast cancer with a radiolabeled antibody Fab fragment. Eur J Nucl Med Mol Imaging: Mar 24, 2015. (Epub ahead of print). doi: 10.1007/s00259-015-3038-1.

30. Saito Y, Hashimoto Y, Kuroda J, Yasunaga M, Koga Y, Takahashi A and Matsumura Y: The inhibition of pancreatic cancer invasion-metastasis cascade in both cellular signal and blood coagulation cascade of tissue factor by its neutralisation antibody. Eur J Cancer 47: 2230-2239, 2011.

31. Matsumura $Y$ and Maeda H: A new concept for macromolecular therapeutics in cancer chemotherapy: Mechanism of tumoritropic accumulation of proteins and the antitumor agent smancs. Cancer Res 46: 6387-6392, 1986.

32. Gurbaxani B, Dostalek M and Gardner I: Are endosomal trafficking parameters better targets for improving $\mathrm{mAb}$ pharmacokinetics than FcRn binding affinity? Mol Immunol 56: 660-674, 2013.

33. Schneider DW, Heitner T, Alicke B, Light DR, McLean K, Satozawa N, Parry G, Yoo J, Lewis JS and Parry R: In vivo biodistribution, PET imaging, and tumor accumulation of $86 \mathrm{Y}-$ and ${ }^{111}$ In-antimindin/RG-1, engineered antibody fragments in LNCaP tumor-bearing nude mice. J Nucl Med 50: 435-443, 2009.

34. Adams GP, Schier R, McCall AM, Simmons HH, Horak EM, Alpaugh RK, Marks JD and Weiner LM: High affinity restricts the localization and tumor penetration of single-chain fv antibody molecules. Cancer Res 61: 4750-4755, 2001.

35. Olafsen T and Wu AM: Antibody vectors for imaging. Semin Nucl Med 40: 167-181, 2010.

36. Kampmeier F, Williams JD, Maher J, Mullen GE and Blower PJ: Design and preclinical evaluation of a $99 \mathrm{mTc}$-labelled diabody of $\mathrm{mAb}$ J591 for SPECT imaging of prostate-specific membrane antigen (PSMA). EJNMMI Res 4: 13, 2014. 